\title{
Hypergeometric Solutions to an Ultradiscrete Painlevé Equation
}

Christopher M. Ormerod

To cite this article: Christopher M. Ormerod (2010) Hypergeometric Solutions to an Ultradiscrete Painlevé Equation, Journal of Nonlinear Mathematical Physics 17:1, 87102, DOI: https://doi.org/10.1142/S140292511000060X

To link to this article: https://doi.org/10.1142/S140292511000060X

Published online: 04 January 2021 


\title{
HYPERGEOMETRIC SOLUTIONS TO AN ULTRADISCRETE PAINLEVÉ EQUATION
}

\author{
CHRISTOPHER M. ORMEROD \\ Department of Mathematics and Statistics \\ La Trobe University \\ Bundoora Victoria 3086 \\ Australia \\ christopher.ormerod@gmail.com
}

Received 2 April 2009

Accepted 13 July 2009

\begin{abstract}
We show that an ultradiscrete analogue of the third Painlevé equation admits discrete Riccati type solutions. We derive these solutions by considering a framework in which the ultradiscretization process arises as a restriction of a non-archimedean valuation over a field. Using this framework we may relax the conditions one requires to apply the ultradiscretization process. We derive a family of transcendental solutions that appear as the non-archimedean field valuation of hypergeometric functions.
\end{abstract}

Keywords: Painlevé; discrete; ultradiscrete; hypergeometric; piece-wise linear; integrable; tropical.

\section{Introduction}

The study of integrable discrete versions of the Painlevé equations is an area of active research [14]. Analogous to the continuous Painlevé equations the discrete Painlevé equations admit rational solutions [10] and also hypergeometric solutions [11]. Ultradiscrete systems are obtained via a limiting process called ultradiscretization [22]. The ultradiscretization process sends a rational function, $f\left(a_{1}, \ldots, a_{n}\right)$, to a new function in a new set of ultradiscrete variables, related by $a_{i}=e^{A_{i} / \epsilon}$, to the limit

$$
F\left(A_{1}, \ldots, A_{n}\right)=\lim _{\epsilon \rightarrow 0} \epsilon \log \left(f\left(a_{1}, \ldots, a_{n}\right)\right) .
$$

This process successfully related an integrable cellular automata, known as the box-ball system [20], to a discrete integrable system [22]. Roughly speaking, it is a transformation that sends binary operations to their ultradiscrete equivalent, given by

$$
\begin{aligned}
a+b & \rightarrow \max (A, B) \\
a b & \rightarrow A+B \\
a / b & \rightarrow A-B,
\end{aligned}
$$


where there is no analog of subtraction. The absence of an ultradiscrete version of subtraction means that the set of functions one may apply the ultradiscretization process to is semifield of functions constructible without the use of the subtraction operation. Many of the methods for studying the resulting equations come from subtraction free methods associated with equations they are derived from [21].

One derives ultradiscrete Painlevé equations by applying the ultradiscretization process to subtraction free versions of the $q$-difference analogs of the Painlevé equations [3]. There are ultradiscrete analogs of all six Painlevé equations [15]. We will focus on the ultradiscretization of the equation

$$
w(q x) w(x / q)=\frac{a_{3} a_{4}\left(w(x)+a_{1} x\right)\left(w(x)+a_{2} x\right)}{\left(w(x)+a_{3}\right)\left(w(x)+a_{4}\right)},
$$

which is given by

$$
\begin{aligned}
\bar{W}+\underline{W}= & A_{3}+A_{4}+\max \left(W, A_{1}+X\right)+\max \left(W, A_{2}+X\right) \\
& -\max \left(W, A_{3}\right)-\max \left(W, A_{4}\right),
\end{aligned}
$$

where $W=W(X), \bar{W}=W(X+Q), \underline{W}=W(X)=W(X-Q)$ and $Q$ is a fixed positive rational number. These equations are known as $q$ - $\mathrm{P}_{\mathrm{III}}$ and $u$ - $\mathrm{P}_{\mathrm{III}}$ respectively.

As the ultradiscretization procedure related a known integrable cellular automata to a known integrable lattice equation [22], the process is said to preserve integrability [15]. This line of reasoning requires one to explore what it means for a system over the max-plus semifield to be integrable. Some indicators of integrability are known to possess a natural embedding in the semifield setting. Indicators include the existence of a Lax Pair $[18,8]$ and singularity confinement [9].

In addition to these integrability indicators, a recent article on an ultradiscrete analogue of Nevanlinna theory has suggested an integrability criterion based on the existence of finite order meromorphic solutions [4]. This work suggests that the ultradiscrete analogue of the a rational function is a function that may be expressed in terms of a finite sequence of the operations of $\max ,+$ and - . Conversely, the ultradiscrete analogue of a transcendental function can be interpreted to be a function that may not be expressed in this way. We find this definition appropriate.

In the above sense, ultradiscrete equations are known to possess rational solutions [21]. These rational solutions come as ultradiscretized subtraction free rational solutions to the $q$-difference equations. An alternate ultradiscretization procedure, known as the sultradiscretization [5], has been utilized to find transcendental solutions to max-plus equations, however, these resulting max-plus versions of the Painlevé equations are of the form max-equals-max. For this reason, we consider the equations resulting from the sultradiscretization procedure to be in a different class to those obtained from the more conventional ultradiscretization. To the authors knowledge, no transcendental solutions of any of the ultradiscrete Painlevé equations have been found.

We present a single example of a hypergeometric solution to an ultradiscrete Painlevé equation. This proves that it is possible to consider solutions that do not appear directly from subtraction free solutions of the $q$-Painlevé equations. However, to achieve this, we are required to redefine ultradiscretization in a way in which it makes sense to take the ultradiscretization of a function that is not necessarily subtraction-free. 
Using a field, $\mathbb{K}$, with a non-archimedean valuation, $\nu$, we lift the discrete Painlevé equation to equations over $\mathbb{K}$, in which $\nu$ acts as a homomorphism of a subsemiring of $\mathbb{K}$, but also naturally extends the ultradiscretization process. As an application of this extension, we apply the valuation to the lift of a hypergeometric solution of (0.4) which is known to map faithfully to a solution of the ultradiscrete equation.

In Sec. 1, we introduce the max-plus semiring and review some key concepts such as valuation rings. We also construct the main tool, the field $\mathbb{K}$. In Sec. 2 we derive solutions of (0.3) by considering Birkhoff's fundamental solutions to the linearization of (0.3). We also highlight some of the problems in applying the ultradiscretization process to these solutions. In Sec. 3, by applying the ultradiscrete analog of Birkhoff theory we derive ultradiscrete fundamental solutions over $\mathbb{K}$ which map faithfully through the valuation to solutions of (0.4). We also discuss the possibility of expressing these solutions in terms of ultradiscrete Bessel functions.

\section{Algebraic Tools}

As mentioned above, we consider the lift of an equation to a field. The choice of valuation field depends on the application. In a previous paper, we used the lifting of linear systems to a valuation field to derive some results regarding the solutions of linear systems over the tropical semiring $S$ [17]. We shall give a standard definition of the max-plus semiring, $S$, then specify the non-archimedean valuation field that we will use.

We let $S=\mathbb{R} \cup\{-\infty\}$, where $S$ possesses the natural ordering on $\mathbb{R}$ and $-\infty$ is the unique minimal element. We equip $S$ with operations of $\oplus$ and $\otimes$, which are defined by

$$
\begin{aligned}
& a \oplus b=\max (a, b) \\
& a \otimes b=a+b .
\end{aligned}
$$

These operations are called tropical addition and tropical multiplication respectively. The 0 element plays the role of the multiplicative identity while $-\infty$ plays the role of the additive identity. We may extend this definition to the set of matrices over $S$ by defining matrix operations $\oplus$ and $\otimes$. If we let $A=\left(a_{i j}\right)$ and $B=\left(b_{i j}\right)$, we may define matrix operations

$$
\begin{aligned}
& {[A \otimes B]_{i j}=\max _{k}\left(a_{i k}+b_{k j}\right)} \\
& {[A \oplus B]_{i j}=\max \left(a_{i j}, b_{i j}\right)}
\end{aligned}
$$

with a scalar multiplication defined by the expression

$$
[\lambda \otimes A]_{i j}=a_{i j}+\lambda .
$$

The ultradiscretization of rational expressions and matrices can be seen as a mapping that preserves addition, multiplication and division. Another way in which one may derive an expression over the max-plus semiring is to consider a non-archimedean valuation ring or field.

Definition 1. A valuation ring is a ring, $R$, with a valuation, $\nu: R \rightarrow \mathbb{R} \cup\{-\infty\}$, such that

(1) $\nu(x)=-\infty$ if and only if $x=0$.

(2) $\nu(x y)=\nu(x)+\nu(y)$.

(3) $\nu(x+y) \leq \nu(x)+\nu(y)$. 
We call a valuation non-archimedean if it has the property

$$
\nu(x+y) \leq \max (\nu(x), \nu(y)) .
$$

Any such ring is metrizable with a metric, $d$, defined as follows

$$
d(x, y)=e^{\nu(x-y)} .
$$

We now employ the well established methods of tropical geometry [16] to extend our ultradiscretization process. Consider the field, $\mathbb{K}$, of algebraic functions in a formal variable $t$, which we write as

$$
\mathbb{K}=\bigcup_{d=1}^{\infty} \mathbb{C}\left(\left(t^{\frac{1}{d}}\right)\right)
$$

We may represent an element, $\phi \in \mathbb{K}$, by its Puiseux series, which is of the form

$$
\phi=a_{0} t^{x_{0}}+a_{1} t^{x_{1}}+\cdots,
$$

where the $a_{i} \in \mathbb{C} \backslash\{0\}, x_{i} \in \mathbb{Q}$ and $x_{i}<x_{i+1}$ for all $i \in \mathbb{N}$. Let $\iota: \mathbb{K} \rightarrow \mathbb{R} \cup\{\infty\}$ be the function that sends $\phi$ to $x_{0}$. In the language of $[16], \iota(\phi)$ is called the order of $\phi$. The non-archimedean valuation, $\nu: \mathbb{K} \rightarrow S$, is defined by

$$
\nu(\phi)=-\iota(\phi)
$$

It should be clear that this mapping is a non-archimedean valuation.

By applying Krull's Existence Theorem [19] we may extend this valuation to the various field extensions of $\mathbb{K}$, as specified in [16]. By applying Krull's Existence Theorem to the algebraic closure of the topological closure of the algebraic closure, one obtains a nonarchimedean valuation field that is both algebraically and topologically closed [19].

To extend the ultradiscretization procedure, we consider the variables, $a_{1}, \ldots, a_{n}$, of a rational function, $f\left(a_{1}, \ldots, a_{n}\right)$, to be functions of $t$. A canonical choice of lift to the algebraic functions would be to let

$$
a_{i}=t^{-A_{i}}
$$

where $A_{i} \in \mathbb{Q}$, which we call the standard lift. We also note that if we restrict $\nu$ to a special subsemiring given by

$$
\mathbb{K}_{0}=\left\{\sum_{i \in \mathbb{N}} u_{i} t^{x_{i}}: u_{i} \in \mathbb{R}^{+} \text {and } x_{i}<x_{i+1} \text { for all } i \in \mathbb{N}\right\}
$$

then $\nu$ acts as a homomorphism of semirings. It should be noted that the action of the valuation $\nu$ and techniques used over $\mathbb{K}_{0}$ mimics the subtraction free nature of the ultradiscretization process.

Lemma 1. Given a rational subtraction free rational function, $f\left(a_{1}, \ldots, a_{n}\right)$, then

$$
\lim _{\epsilon \rightarrow 0} \epsilon \log f\left(e^{\frac{A_{1}}{\epsilon}}, \ldots, e^{\frac{A_{n}}{\epsilon}}\right)=\nu\left(f\left(t^{-A_{1}}, \ldots, t^{-A_{n}}\right)\right) .
$$

The standard lift is a canonical choice of lift, but it is worth noting that one tool of this theory is to consider liftings that are not standard, such as $a \rightarrow t^{-A}+E$ where 
$\nu\left(t^{-A}+E\right)=A$. As $\nu$ is a mapping over all of $\mathbb{K}$, the ultradiscretization may be viewed as a restriction of a non-archimedean valuation. When ultradiscretization is viewed in this manner, the connection between tropical algebraic geometry [16] and ultradiscrete equations follows more naturally.

Although the valuation naturally extends the ultradiscretization process, to apply this extended ultradiscretization to integrable systems over the max-plus semifield one requires conditions in which equality hold in (1.2). A simple lemma of the theory of non-archimedean valuation fields gives us a partial answer:

Lemma 2. If $\nu(f(t)) \neq \nu(g(t))$, then $\nu(f(t) \pm g(t))=\max (\nu(f(t)), \nu(g(t)))$.

This will be sufficient for a class of solutions considered.

\section{Derivation of $q$-Hypergeometric Solutions}

The continuous Painlevé equations admit particular solutions that may be expressed in terms of Gauss' hypergeometric functions and it is generalizations. In the case of $q$ difference equations, the $q$-analogue of Gauss' hypergeometric function is Heine's hypergeometric function [1], also known as the basic hypergeometric function or $q$-hypergeometric function, given by

$$
{ }_{2} \phi_{1}\left(\begin{array}{c}
a, b \\
c
\end{array} ; q, x\right)=\sum_{m=0}^{\infty} x^{m} \frac{(a, b ; q)_{m}}{(c, q ; q)_{m}}
$$

where

$$
\left(a_{1}, \ldots, a_{n} ; q\right)_{k}=\left(a_{1} ; q\right)_{k} \cdots\left(a_{n} ; q\right)_{k}
$$

and

$$
(a ; q)_{k}=(1-a)(1-a q) \cdots\left(1-a q^{k-1}\right)
$$

is the $q$-Pochhammer symbol.

We begin by examining a version of $q-\mathrm{P}_{\text {III }}$ in which the hypergeometric solutions are known,

$$
\bar{w} \underline{w}=\frac{a_{3} a_{4}\left(w+a_{1} x\right)\left(w+a_{2} x\right)}{\left(w+a_{3}\right)\left(w+a_{4}\right)}
$$

where $w=w(x), \bar{w}=w(q x)$ and $\underline{w}=w(x / q)$. This system was introduced in [14] and its hypergeometric solutions were obtained by the use of the $q$-Bessel function in [2]. This equation possesses an associated linear problem [2], or Lax pair, and is equivalent to a special case of $q-\mathrm{P}_{\mathrm{VI}}[7]$. The equation is known to admit special solutions expressible in terms of basic hypergeometric functions. By considering the discrete analogue of the Riccati equation (the fractional linear transformation), given by

$$
\bar{w}=\frac{\alpha w+\beta}{\gamma w+\delta} .
$$


Solving for $w$ in terms of $\bar{w}$ gives

$$
\underline{w}=\frac{\underline{\delta} w-\underline{\beta}}{-\underline{\gamma} w+\underline{\alpha}} .
$$

At this point, we see that if the parameters of (2.3) are subtraction free then (2.4) is not subtraction free in general.

The condition $a_{2}=\frac{q a_{1} a_{3}}{a_{4}}$ is required so that (2.3) and (2.4) coincide with (2.2). Assuming this condition means that (2.2) may be linearized into

$$
\bar{w}=-\frac{q x a_{1} a_{3}+a_{4} w}{a_{3}+w},
$$

where letting a $x \rightarrow x / q$ and solving for $w$ reveals that the first order equation defining the backwards evolution is

$$
\underline{w}=-\frac{x a_{1} a_{3}+a_{3} w}{a_{4}+w} .
$$

At this point, it becomes clear that any solution derived by this method cannot be made subtraction free.

We make the substitution $w=s y$ where $s=\sqrt{x}$ and $p=\sqrt{q}$ to derive the equation

$$
\bar{y}=-\frac{p^{2} s a_{1} a_{3}+a_{4} y}{p a_{3}+p s y}
$$

By using the Cole-Hopf transformation, $y=u / v$, we reduce this to the linear system

$$
\begin{gathered}
\left(\begin{array}{l}
\bar{u} \\
\bar{v}
\end{array}\right)=\left(\left(\begin{array}{cc}
-a_{4} & 0 \\
0 & p a_{3}
\end{array}\right)+\left(\begin{array}{cc}
0 & -p^{2} a_{1} a_{3} \\
p & 0
\end{array}\right) s\right)\left(\begin{array}{l}
u \\
v
\end{array}\right) \\
\bar{\Psi}=\left(A_{0}+A_{1} s\right) \Psi .
\end{gathered}
$$

Since $A_{0}$ is diagonal, this is done by a substitution of

$$
\Psi(s)=\left(I+Y_{1} s+Y_{2} s^{2}+\cdots\right) A_{0}^{\log _{p} s}
$$

into the linear equation to obtain a recurrence relation for the sequence $Y_{k}$. The terms $Y_{k}$ may be solved in terms of $q$-Pochhammer symbols. However, explicit solutions in terms of ${ }_{2} \phi_{1}$ functions can also be obtained via an application of the work of LeCaine [13]. This shows the fundamental solution around 0 of (2.8) are

$$
\begin{aligned}
Y(x, t)= & \Gamma_{p}\left(1+\frac{t}{a_{1} a_{3} p}\right)\left(\begin{array}{cc}
\Phi_{1}(s) & \Phi_{2}(s) \\
\frac{\Phi_{1}(p s)}{\sqrt{a_{1} a_{4}}} & \frac{\Phi_{2}(p s)}{a_{3} p} \sqrt{\frac{a_{4}}{a_{1}}}
\end{array}\right) \\
& \times \operatorname{diag}\left(\left(-a_{4} / p\right)^{\log _{p} s}, a_{3}^{\log _{p} s}\right),
\end{aligned}
$$


where the $\Phi$ functions are given by

$$
\begin{gathered}
\Phi_{1}(s)={ }_{2} \phi_{1}\left(\begin{array}{c}
\left.\frac{1}{\sqrt{a_{1} a_{4}}}, a_{3} \sqrt{\frac{q a_{1}}{a_{4}}} ; p, s\right) \\
q \frac{a_{3}}{a_{4}}
\end{array}\right) \\
\Phi_{2}(s)={ }_{2} \phi_{1}\left(\begin{array}{c}
\frac{1}{a_{3}} \sqrt{\frac{a_{4}}{q a_{2}}}, \sqrt{a_{1} a_{4}} \\
\frac{a_{4}}{a_{3}}
\end{array} p, s\right),
\end{gathered}
$$

and $\Gamma_{q}$ is the $q$-gamma function in [12]. This calculation may be used to express a special solution of (0.3) explicitly in terms of $\Phi_{1}$ and $\Phi_{2}$. Furthermore, the first order equation defining the forwards and backwards evolution, namely (2.5) and (2.6), cannot be made subtraction free simultaneously. Nor can the solutions of (2.5), since they are expressed in terms of $q$-Pochhammer symbols.

We have provided one approach that relies on the theory of linear $q$-difference equations, which we will adapt to the ultradiscrete setting the next section. Another approach uses the Cole-Hopf transformation, $w=u / v$, applied to (2.5) to derive a second order difference equation for $v$, which is identified with the $q$-Bessel function. Details of this method can be found in $[2,11]$.

\section{Ultradiscrete Hypergeometric Solutions}

The question we wish to address is whether transcendental solutions exist for (0.4). We shall answer this question in the affirmative by providing a single family of examples. We shall demonstrate how one may find an explicit form of the solutions by using an ultradiscrete analogue of the theory of the previous section.

If we consider basic ultradiscrete hypergeometric function to be the application of the valuation to the basic hypergeometric function, after applying the standard lift $q \rightarrow t^{-Q}$, $a \rightarrow t^{-A}, b \rightarrow t^{-B}, c \rightarrow t^{-C}$ and $x \rightarrow t^{-X}$, we obtain the function

$$
\begin{aligned}
{ }_{2} \Phi_{1}\left(\begin{array}{c}
A, B \\
C
\end{array} ; Q, X\right)= & \max _{m \in \mathbb{N}}\left(m X+m\left(\max \left(A,-\frac{m-1}{2} Q\right)+\max \left(B,-\frac{m-1}{2} Q\right)\right.\right. \\
& \left.\left.-\max \left(C,-\frac{m-1}{2} Q\right)-\max \left(Q,-\frac{m-1}{2} Q\right)\right)\right),
\end{aligned}
$$

which converges when $X<C+Q-A-B$. In the cases that present themselves in this work, either $A$ or $B$ will be $-\infty$, hence, the hypergeometric functions considered here will converge everywhere. The case in which either $A$ or $B$ are $-\infty$ is interesting because the hypergeometric function is discontinuous at an infinite number of $X$ values, hence, represents a truly transcendental ultradiscrete function.

The ultradiscretization method applied to (2.2) gives us the discrete dynamical system

$$
\begin{aligned}
\bar{W}+\underline{W}= & A_{3}+A_{4}+\max \left(W, A_{1}+X\right)+\max \left(W, A_{2}+X\right) \\
& -\max \left(W, A_{3}\right)-\max \left(W, A_{4}\right) .
\end{aligned}
$$

This equation is known to possess rational solutions such as those obtained via orbits of Bäcklund transformations [15] applied to the solution $W=X / 2$ for $A_{1}=A_{2}=A_{3}=A_{4}=$ 0. Subtraction free versions of the transformations found in [6] may be ultradiscretized to 
form a representation of the affine Weyl group of type $A_{1}^{(1)} \times A_{1}^{(1)}$ as a group of Bäcklund transformations. By considering the orbit of this group, we find the set of rational solutions of $(0.4)$.

We lift (0.3) to $\mathbb{K}$ via the standard lift. That is to say we lift the constants $a_{i} \rightarrow t^{-A_{i}}$, $x \rightarrow t^{-X}, q \rightarrow t^{-Q}$ and $W \rightarrow \mathscr{W}$, where the only requirement is that $\mathscr{W}$ be a function of $t$. The lifting gives the equation

$$
\overline{\mathscr{W}} \underline{\mathscr{W}}=\frac{t^{-A_{3}} t^{-A_{4}}\left(\mathscr{W}+t^{-A_{1}-X}\right)\left(\mathscr{W}+t^{-A_{2}-X}\right)}{\left(\mathscr{W}+t^{-A_{3}}\right)\left(\mathscr{W}+t^{-A_{4}}\right)} .
$$

If $\mathscr{W}$ is an element of $\mathbb{K}_{0}$, then we have that the valuation, $\nu$, brings (3.2) to (0.4). For general $\mathscr{W}$ however, we have a set of inequalities obtained by breaking up the parts. Given $\nu(\mathscr{W})=W$, we have the following list of inequalities

$$
\begin{aligned}
\nu\left(\mathscr{W}+t^{-A_{1}-X}\right) & \leq \max \left(W, A_{1}+X\right) \\
\nu\left(\mathscr{W}+t^{-A_{2}-X}\right) & \leq \max \left(W, A_{2}+X\right) \\
\nu\left(\mathscr{W}+t^{-A_{3}}\right) & \leq \max \left(W, A_{3}\right) \\
\nu\left(\mathscr{W}+t^{-A_{4}}\right) & \leq \max \left(W, A_{4}\right) .
\end{aligned}
$$

If it is possible to show the equality for each of these expressions for all $X$, then we have a solution. For example, if $\mathscr{W}$ was a subtraction free rational expression, then equality would hold. Alternatively, if $\mathscr{W}$ may be expressed as an addition of terms in which the dominant term was unique, then Lemma 2 specifies that equality holds.

A further relaxation to having the equality hold in each application of the valuation is that equality may not hold for each term, however, the amount that they differ cancels out so that the resulting max-plus expression still holds. For example, consider

$\nu\left((1-a) t^{-A}+t^{-A+1}\right)-\nu\left((1-a) t^{-B}+t^{-B+1}\right)=\max (A, A-1)-\max (B, B-1)=A-B$.

Even when $a=1$, where the $t^{-A}$ and $t^{-B}$ terms do not appear in the Puiseux expansion, equality still holds because the differences between the valuation and the max expressions cancel out.

Generally speaking, this method considers the possibility of considering this equality outside of $\mathbb{K}_{0}$. That is to say that it is possible to solve max-plus equations from expressions that are not necessarily subtraction free.

As an example, let us consider the linearization of (3.2). We should be able to derive solutions in a similar manner to the work above for (2.2). We substitute the following lifted ultradiscretization of a discrete analogue of the Riccati equation over $\mathbb{K}$

$$
\overline{\mathscr{W}}=\frac{t^{\alpha}+t^{\beta} \mathscr{W}}{t^{\gamma}+t^{\delta} \mathscr{W}}
$$

We find conditions for (3.4) to describe the evolution of (3.2). These conditions coincide with the ultradiscretized conditions for the linearization of (2.2) for similar reasons. These 
are that $A_{2}=Q+A_{1}+A_{3}-A_{4}$, giving the linear system over $\mathbb{K}$

$$
\begin{aligned}
& \overline{\mathscr{W}}=\frac{-t^{-A_{1}-A_{3}-X-Q}-t^{-A_{4}} \mathscr{W}}{t^{-A_{3}}+\mathscr{W}} \\
& \underline{\mathscr{W}}=\frac{-t^{-A_{1}-A_{3}-X}-t^{-A_{3}} \mathscr{W}}{t^{-A_{4}}+\mathscr{W}} .
\end{aligned}
$$

The inequalities for (3.5a) are

$$
\begin{aligned}
\nu\left(-t^{-A_{1}-A_{3}-X-Q}-t^{-A_{4}} \mathscr{W}\right) & \leq A_{4}+\max \left(A_{2}+X, W\right) \\
\nu\left(t^{-A_{3}}+\mathscr{W}\right) & \leq \max \left(A_{3}, W\right) .
\end{aligned}
$$

For $(3.5 \mathrm{~b})$ we have

$$
\begin{aligned}
\nu\left(-t^{-A_{1}-A_{3}-X}-t^{\left.-A_{3} \mathscr{W}\right)}\right. & \leq A_{3}+\max \left(A_{1}+X, W\right) \\
\nu\left(t^{-A_{4}}+\mathscr{W}\right) & \leq \max \left(A_{4}, W\right)
\end{aligned}
$$

where in each case, if (3.3) holds for (3.6) and (3.7), then $\nu(\mathscr{W})$ is a solution of (0.4). Given appropriate conditions, it is possible that this equality holds for all time. If equality does hold, then the forward and backward evolution is given simply by two ultradiscrete Riccati type equations

$$
\begin{aligned}
& \bar{W}=\max \left(A_{1}+A_{3}+X+Q, A_{4}+W\right)-\max \left(A_{3}, W\right) \\
& \underline{W}=\max \left(A_{1}+A_{3}+X, A_{3}+W\right)-\max \left(A_{4}, W\right) .
\end{aligned}
$$

Equation (3.8a) can be seen as the ultradiscretization of a version of (2.3) in which all the parameters are subtraction free. If we do this, the relevant backwards evolution, defined by (2.4), would not be subtraction free, meaning we could not apply a conventional ultradiscretization to obtain (3.8b). Hence, any function solving (3.8a) and (3.8b) simultaneously (and hence (0.4)) cannot come from a subtraction free solution of (2.3) (and (2.4)).

Figure 1 shows two examples of solutions of (0.4) satisfying both (3.8) and (3.8b).

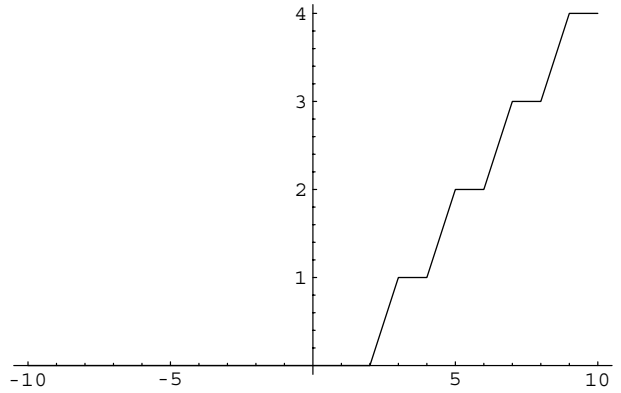

(a) Hypergeometric solution for parameters $A_{1}=-2$, $A_{2}=-1, A_{3}=0, A_{4}=0, Q=1$ and $W_{0}(0)=0$.

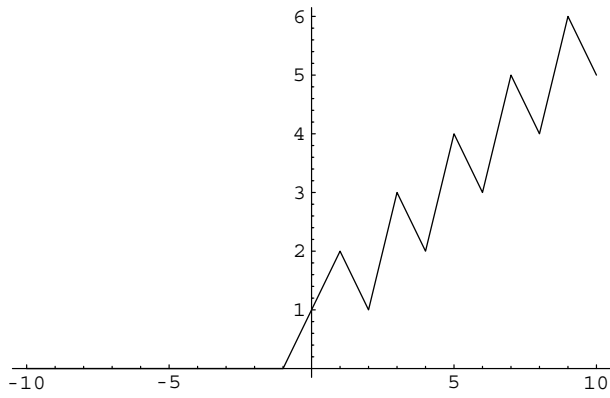

(b) Hypergeometric solution for parameters $A_{1}=0$, $A_{2}=2, A_{3}=1, A_{4}=0, Q=1$ and $W_{0}(0)=1$.

Fig. 1. 2 Hypergeometric solutions to (0.4). 
One particular family of systems generalizing of the behavior seen in Fig. 1(a) is defined by the conditions

$$
\begin{gathered}
A_{1}=-(r+1) Q \quad A_{2}=Q+A_{1}+A_{3}-A_{4}=-r Q \\
A_{3}=0 \quad A_{4}=0
\end{gathered}
$$

where $W(0)=0$ and $r>1$. We will call this particular example a hypergeometric solution of (0.4). From here on, we shall be considering this example in detail from various standpoints.

The first standpoint is one in which we stay within the confines of the semifield setting. We note that any solution of (0.4) may satisfy inequalities that reduce the evolution to a sequence of linear difference equations on different intervals.

For $X<r Q<(r+1) Q$, a solution where $W \geq 0$ means that the right-hand side of $(0.4)$ is 0 and the left-hand side of $(0.4)$ is $\bar{W}+\underline{W}$. This is solved by $W=0$. Also for $W \geq 0$, the right-hand side of (3.8) is $W$, while the left-hand side is $\bar{W}$. This is also solved by $W=0$.

We make the ansatz that for large positive $X$, the behavior is proportional to $X$, as seen in Fig. 1. Balancing the large $X$ behavior of $W$ reveals 3 separate cases; $W \sim k X$ for $k>1, k<0$ and $0<k<1$. Each of these cases specify what terms dominate in the $\max$ terms in (0.4) and (3.8). The first specifies that $W$ dominates in all the max terms, leading to $\bar{W}+\underline{W} \sim 2 k X$ on the left-hand side of (0.4) and on the right-hand side is asymptotic to some constant, which is a contradiction. For $k<0$, on the left-hand side of (0.4), $\bar{W}+\underline{W} \sim 2 k X$ and on the right-hand side we have $2 X$, but $k=1$ contradicts the assumptions. Lastly, we have that where $0<k<1$, on the right-hand side of (0.4), $A_{1}+X$ and $A_{2}+X$ dominate in the first two max terms, while the $W$ dominates in the remaining max terms. The resulting linear difference equation $W$ solves for large $X$ is

$$
\bar{W}+\underline{W}=A_{3}+A_{4}+A_{1}+A_{2}+2 X-2 W .
$$

The general solution of this second order difference equation is of the form

$$
W=\frac{X}{2}+\frac{A_{1}+A_{2}+A_{3}+A_{4}}{4}+a(-1)^{X / Q}+\frac{b X}{Q}(-1)^{X / Q}
$$

where $-Q / 2<b<Q / 2$ by the asymptotic assumptions imposed. The above logic also applies to (3.8). This linear difference equation approach is consistent with the behavior seen in figure. Particular choices of $r$ result in different choices of $a$ and $b$. One only needs to take into account what the transient behavior is around $X=(r+1) Q$.

Given a solution where the evolution governed by both (0.4) and (3.8), we consider the lifted equation, (3.5), as also having a solution defined over $\mathbb{K}$, which may be considered an analog of the hypergeometric function over a field. We shall be able to derive explicit expressions for our new ultradiscrete hypergeometric functions through this correspondence and $\nu$. Since the evolution is governed by (3.5), we can use a Cole-Hopf transformation over $\mathbb{K}$. By substituting $\mathscr{W}=U / V$, we derive the linear system associated with Fig. 1(a). From (3.5), with our particular choice of variables, we have the linear system

$$
\left(\begin{array}{l}
\bar{U} \\
\bar{V}
\end{array}\right)=\left(\begin{array}{cc}
-1 & -t^{r Q-X} \\
1 & 1
\end{array}\right)\left(\begin{array}{l}
U \\
V
\end{array}\right)=Y\left(t^{-X-Q}\right)=B\left(t^{-X}\right) Y\left(t^{-X}\right)
$$


where $B\left(t^{-X}\right)=B_{0}+B_{1} t^{-X}$ and

$$
B_{0}=\left(\begin{array}{cc}
-1 & 0 \\
1 & 1
\end{array}\right), \quad B_{1}=\left(\begin{array}{cc}
0 & -t^{r Q} \\
0 & 0
\end{array}\right) .
$$

This is a linear system in which the framework of [17] applies. We may solve this explicitly by substituting an expression of the form

$$
\begin{aligned}
Y\left(t^{-X}\right) & =\left(Y_{0}+Y_{1} t^{-X}+Y_{2} t^{-2 X}+\cdots\right) \operatorname{diag}(-1,1)^{\frac{X}{Q}} \\
& =\hat{Y}\left(t^{-X}\right) D_{0}^{X / Q}
\end{aligned}
$$

which we substitute into (3.10). By equating the constant coefficient of $\hat{Y}$ we have

$$
Y_{0} D_{0}=B_{0} Y_{0}
$$

hence $Y_{0}$ diagonalizes $A_{0}$. We let

$$
Y_{0}=\left(\begin{array}{cc}
2 & 0 \\
-1 & 1
\end{array}\right)
$$

For the rest of the power series, we derive the recursion relation

$$
t^{-k Q} Y_{k} D_{0}-B_{0} Y_{k}=B_{1} Y_{k-1}
$$

If we let $Y_{k}=y_{i j}^{(k)}$, then we may derive the recursive relation for each of components. These relations are

$$
\begin{aligned}
y_{11}^{(k)} & =-\frac{t^{r Q} y_{21}^{(k-1)}}{\left(1-t^{-k Q}\right)} \\
y_{12}^{(k)} & =-\frac{t^{r Q} y_{22}^{(k-1)}}{\left(1+t^{-k Q}\right)} \\
y_{21}^{(k)} & =-\frac{y_{11}^{(k)}}{\left(1+t^{-k Q}\right)} \\
& =\frac{t^{r Q} y_{21}^{(k-1)}}{1-t^{-2 k Q}} \\
y_{22}^{(k)} & =-\frac{y_{12}^{(k)}}{\left(1-t^{-k Q}\right)} \\
& =\frac{t^{r Q} y_{22}^{(k-1)}}{1-t^{-2 k Q}} .
\end{aligned}
$$

The relations for $y_{21}^{(k)}$ and $y_{22}^{(k)}$ are solved explicitly by

$$
\begin{aligned}
y_{21}^{(k)} & =-\frac{t^{k r Q}}{\left(t^{-Q} ; t^{-Q}\right)_{k}\left(-t^{-Q} ; t^{-Q}\right)_{k}} \\
y_{22}^{(k)} & =\frac{t^{k r Q}}{\left(t^{-Q} ; t^{-Q}\right)_{k}\left(-t^{-Q} ; t^{-Q}\right)_{k}} .
\end{aligned}
$$


Solving this recursion relation, we arrive at the coefficients

$$
Y_{k}=\left(t^{r Q}\right)^{k}\left(\begin{array}{cc}
\frac{1+t^{-k Q}}{\left(t^{-Q} ; t^{-Q}\right)_{k}\left(-t^{-Q} ; t^{-Q}\right)_{k}} & \frac{-\left(1-t^{-k Q}\right)}{\left(t^{-Q} ; t^{-Q}\right)_{k}\left(-t^{-Q} ; t^{-Q}\right)_{k}} \\
\frac{-1}{\left(t^{-Q} ; t^{-Q}\right)_{k}\left(-t^{-Q} ; t^{-Q}\right)_{k}} & \frac{1}{\left(t^{-Q} ; t^{-Q}\right)_{k}\left(-t^{-Q} ; t^{-Q}\right)_{k}}
\end{array}\right)
$$

where we have the $q$-Pochhammer symbol over $\mathbb{K}$,

$$
(A ; B)_{k}=(1-A)(1-A B) \cdots\left(1-A B^{k-1}\right) .
$$

If we extend the hypergeometric notation of these objects over $\mathbb{K}$, we let

$$
\Phi\left(t^{-X}\right)={ }_{2} \phi_{1}\left(\begin{array}{c}
0,0 \\
-t^{-Q}
\end{array} ; t^{-Q},-t^{r Q-X}\right)
$$

we may express the solution as

$$
Y\left(t^{-X}\right)=\left(\begin{array}{cc}
\Phi\left(t^{-X-Q}\right)+\Phi\left(t^{-X}\right) & \Phi\left(t^{-X-Q}\right)-\Phi\left(t^{-X}\right) \\
-\Phi\left(t^{-X}\right) & \Phi\left(t^{-X}\right)
\end{array}\right)
$$

We require a choice of initial conditions for $U$ and $V$ such that $\nu(U(0) / V(0))=0$ and $U \neq V$ so that a division by 0 does not occur. By setting the initial condition to be $(U(0), V(0))=\left(t^{0}, 2 t^{0}\right)=(1,2)$, the solution is given by

$$
\mathscr{W}=\frac{3 \Phi\left(t^{-X-Q}\right)-\Phi\left(t^{-X}\right)}{\Phi\left(t^{-X}\right)} .
$$

We calculate the valuation of this solution from (3.11) by noting how the valuation acts on the various parts. In particular, we have

$$
\begin{aligned}
\nu\left(\left(t^{-Q}: t^{-Q}\right)_{k}\right) & =\nu\left(\left(1-t^{Q}\right) \ldots\left(1-t^{-k Q}\right)\right. \\
& =\sum_{j=1}^{k} \nu\left(1-t^{-j Q}\right) \\
& =\sum_{j=1}^{k} \max (0, j Q) \\
& =\sum_{j=1}^{k} j Q=\frac{1}{2} k(k+1) Q
\end{aligned}
$$

and similarly

$$
\nu\left(\left(-t^{-Q}: t^{-Q}\right)_{k}\right)=\frac{1}{2} k(k+1) Q
$$


We may calculate the valuation of (3.13) for each $X$. Firstly, we split up this calculation by noting

$$
\nu(\mathscr{W})=\nu\left(3 \Phi\left(t^{-X-Q}\right)-\Phi\left(t^{-X}\right)\right)-\nu\left(\Phi\left(t^{-X}\right)\right) .
$$

Let us calculate the valuations of $\Phi\left(t^{-X}\right)$ for all $X$ (by considering the bottom right entry of (3.12) and (3.11)). The coefficient of $t^{-k X}$ is

$$
\frac{t^{r k Q}}{\left(t^{-Q} ; t^{-Q}\right)_{k}\left(-t^{-Q} ; t^{-Q}\right)_{k}}
$$

whose valuation is $-k r Q-k(k+1) Q$, for all $k$. Hence, for $X<(r+2) Q,-k r Q-k(k+1) Q<$ $k X$, so the constant coefficient dominates giving $\nu\left(\Phi\left(t^{-X}\right)\right)=0$. Let $X_{k}$ be the point at which the valuation of the $t^{-k X}$ and $t^{-(k+1) X}$ are equal, this is determined by

$$
\nu\left(\frac{t^{-k\left(r Q-X_{k}\right)}}{\left(t^{-Q} ; t^{-Q}\right)_{k}\left(-t^{-Q} ; t^{-Q}\right)_{k}}\right)=\nu\left(\frac{t^{-(k+1)\left(r Q-X_{k}\right)}}{\left(t^{-Q} ; t^{-Q}\right)_{k+1}\left(-t^{-Q} ; t^{-Q}\right)_{k+1}}\right) .
$$

This simple calculation gives $X_{k}=(r+2+2 k) Q$. Since $X_{k}$ is monotonically increasing with $k$ (in integer increments), the $t^{-k Q}$ term must dominate for $(r+2+2 k) Q<X<(r+4+2 k) Q$ uniquely. The uniqueness allows us to use Lemma 2 to give that

$$
\nu\left(\Phi\left(t^{-X}\right)\right)=\max _{k \in \mathbb{N}}(-k r Q-(k+1) k Q+k X)
$$

for $X \notin\left\{X_{k}\right\}$. Hence, this expression holds on a dense subset of $\mathbb{R}$.

This calculation and the action of the valuation of the other parts of (3.11) give the following valuation in terms of the ultradiscrete hypergeometric function

$$
\begin{aligned}
\nu\left(\frac{3 \Phi\left(t^{-X-Q}\right)-\Phi\left(t^{-X}\right)}{\Phi\left(t^{-X}\right)}\right)= & { }_{2} \Phi_{1}\left(\begin{array}{c}
-\infty,-\infty \\
Q
\end{array}\right. \\
& -{ }_{2} \Phi_{1}\left(\begin{array}{c}
-\infty,-\infty \\
Q
\end{array}, Q, X-r Q\right) .
\end{aligned}
$$

The following theorem sums up the major result of this paper.

Theorem 1. The ultradiscrete Painlevé equation given by (0.4) with parameters specified by (3.9) is solved by the function

$$
\begin{aligned}
W= & \max _{k \in \mathbb{N}}\left(-k r Q-k^{2} Q+k X\right) \\
& -\max _{k \in \mathbb{N}}(-k r Q-(k+1) k Q+k X)
\end{aligned}
$$

for all $r>1$.

Proof. We shall now show through elementary means that (3.14) is a solution (0.4) with the appropriate initial conditions. We do this by showing that (3.8) and (3.8b) are satisfied 
simultaneously. We simplify this expression by letting $X=n Q$. Hence, we write

$$
W=Q \max _{k}(k(n-r-k))-Q \max _{k}(k(n-r-k-1)),
$$

where $\bar{W}(n)$ is now $W(n+1)$. Since $A_{3}=0$ and $W \geq 0$ for all $n$, the $W$ in the second $\max$ in (3.8a) dominates meaning (3.8a) simplifies to

$$
\bar{W}=\max (Q(n-r)-W, 0) .
$$

Substituting (3.15) into (3.16) on the left-hand side

$$
\bar{W}=Q \max _{k}(k(n+1-r-k))-Q \max _{k}(k(n-r-k)),
$$

while on the right-hand side of (3.16) we have

$$
\begin{aligned}
\bar{W}= & Q \max \left((n-r)+\max _{k}(k(n-r-k-1))-\max _{k}(k(n-r-k)), 0\right) \\
= & Q \max _{k}((k+1) n-(k+1) r-k(k+1), k(n-r-k)) \\
& -Q \max _{k}(k(n-r-k)) .
\end{aligned}
$$

By shifting the $k$ component in the first part of the right-hand sides max expression, we write

$$
\begin{aligned}
\bar{W} & =Q \max _{k \geq 1}\left(k n-k r-k(k-1), k n-k r-k^{2}, 0\right)-Q \max _{k}(k(n-r-k)) \\
& =Q \max _{k \geq 1}(k(n-r-k+1), k(n-r-k))-Q \max _{k}(k(n-r-k)) .
\end{aligned}
$$

We may simplify the right-hand side by noticing that the first elements in the max always dominates for $k \geq 1$. This gives the equality

$$
\begin{aligned}
\bar{W} & =Q \max _{k \geq 1}\left(k n-k r-k^{2}+k, 0\right)-Q \max _{k}(k(n-r-k)) \\
& =Q \max _{k}(k(n-r-k+1))-Q \max _{k}(k(n-r-k))
\end{aligned}
$$

which shows (3.15) does satisfy (3.8a). To see that this equation satisfies (3.8b), the forward equation holds, so we may de-evolve the forward evolution equation giving

$$
W=\max ((n-r-1) Q-\underline{W}, 0)
$$

where if $W>0$ then in particular 0 must not dominate in the max, giving $W=(n-r-$ 1) $Q-\underline{W}$. We also know that $W \geq \underline{W} \geq 0$. Hence for $W=0, \underline{W}=0$, we have

$$
\underline{W}=\max ((n-r-1) Q-W, 0)
$$

for all $n$. This shows (3.15) coincides with (3.8b) for our choice of $W$ and our parameters. Hence (3.15) solves (3.8). This then immediately implies (3.14) solves u-P $\mathrm{P}_{\mathrm{III}}$.

This shows that the ultradiscrete hypergeometric solution found has an evolution defined by some ultradiscretized discrete Riccati equation and also appears as the valuation of a hypergeometric equation in a higher space. 
An alternative approach to that of the linear systems approach is to consider the these solutions in terms of $q$-Bessel functions similar to that of [11]. Instead of the Cole-Hopf transformation, we may consider a different substitution given by

$$
\mathscr{W}=\frac{\bar{J}}{J}-1 .
$$

Under the condition $t^{-A_{1}}=t^{(r+1) Q}, t^{-A_{3}}=1$ and $t^{-A_{4}}=-1$ then $J$ satisfies the equation

$$
\bar{J}-J(1+1)+\underline{J}\left(1+t^{-X+(r+1) Q}\right)=0
$$

which is then a lifted form of the $q$-Bessel equation for $J_{0}\left(t^{S}\right)$ where $2 S=X$. This is a special case of the $q$-Bessel function $J_{\nu}$, where $\nu=0$, the resultant solution of this equation is the $q$-Bessel function over $\mathbb{K}$. This function simplifies in the case $\nu=0$ to the equation

$$
J_{0}(X ; Q)={ }_{2} \phi_{1}\left(\begin{array}{c}
0,0 \\
t^{-Q}
\end{array} ; t^{-Q}, t^{-X+r Q}\right) .
$$

By substituting back into $\mathscr{W}$, and applying the valuation with appropriate initial conditions, we recover the same formula for the solution as found by our previous method with linear systems.

\section{Conclusion}

This method also opens a door to allow us to consider studies in ultradiscrete equations outside the ultradiscretization of any subtraction free method applied to $q$-difference equations. In particular, it allows us to consider solutions of various ultradiscrete equations in which the solution does not come as an ultradiscretized subtraction free solution of the $q$-difference equation it was derived from. Furthermore, putting ultradiscretization in terms of non-archimedean valuations paves the way for connections with tropical geometry.

Despite the existence of hypergeometric type solutions, the conditions in which the valuation yields equality of both sides is unclear when considering a solution holding for all time, making it difficult to assert when this method will give rise to new solutions. Further investigation into these conditions may give rise to other types of solutions, including the precise conditions for when a ultradiscrete equation has what we consider a hypergeometric solution. In particular, it would be interesting to see whether solutions may be parameterized in terms of a general ultradiscrete Bessel function.

Although this function came as a generalized ultradiscretization of a hypergeometric function, it would be interesting to see what properties they possess. These hypergeometric functions should possess transformation formulas, connection formulas and contiguity relations, and it is an area of research well worth pursuing.

\section{Acknowledgments}

The author would like to acknowledge the contributions of Professor Kenji Kajiwara, Doctor Chris Field, Doctor James Atkinson and Professor Yasuhiko Yamada for their helpful points and insights. I would also like to thank Professor Nalini Joshi for her encouragement in this research. This research was supported in part by the Australian Research Council grant \#DP0559019. 


\section{References}

[1] G. Gasper and M. Rahman, Basic hypergeometric series, with a foreword by Richard Askey, Encyclopedia of Mathematics and its Applications 35 (Cambridge University Press, Cambridge, 1990).

[2] B. Grammaticos, F. W. Nijhoff, V. Papageorgiou, A. Ramani and J. Satsuma, Linearization and solutions of the discrete Painlevé III equation, Phys. Lett. A 185(5-6) (1994) 446-452.

[3] B. Grammaticos, Y. Ohta, A. Ramani, D. Takahashi and K. M. Tamizhmani, Cellular automata and ultra-discrete Painlevé equations, Phys. Lett. A 226(1-2) (1997) 53-58.

[4] R. G. Halburd and N. J. Southall, Tropical Nevanlinna theory and ultradiscrete equations, Int. Math. Res. Not. 5 (2009) 887-911.

[5] S. Isojima, B. Grammaticos, A. Ramani and J. Satsuma, Ultradiscretization without positivity, J. Phys. A 39 (2006) 3663-3672.

[6] M. Jimbo, B. Grammaticos, A. Ramani and H. Sakai, Bilinear structure and Schlesinger transforms of the $q-\mathrm{P}_{\mathrm{III}}$ and $q-\mathrm{P}_{\mathrm{VI}}$, Phys. Lett. A 217(2) (1996) 111-118.

[7] M. Jimbo and H. Sakai, A q-analog of the sixth Painlevé equation, Lett. Math. Phys. 38(2) (1996) 145-154.

[8] N. Joshi, F. Nijhoff and C. M. Ormerod, Lax pairs for ultra-discrete Painlevé cellular automata, J. Phys A: Math. Gen. 37(44) (2004) L559-L565.

[9] N. Joshi and S. Lafortune, How to detect integrability in cellular automata, J. Phys. A 38(28) (2005) L499-L504.

[10] K. Kajiwara, K. Yamamoto and Y. Ohta, Rational solutions for the discrete Painlevé II equation, Phys. Lett. A 232(3-4) (1997) 189-199.

[11] K. Kajiwara, Y. Ohta and J. Satsuma, Casorati determinant solutions for the discrete Painlevé III equation, J. Math. Phys. 36(8) (1995) 4162-4174.

[12] R. Koekoek and R. F. Swarttouw, The Askey-scheme of hypergeometric orthogonal polynomials and it's q-analogue, Report 94-05, Delft University of Technology, Faculty TWI (1994).

[13] J. Le Caine, The linear q-difference equation of the second order, Amer. J. Math. 65 (1943) 585-600.

[14] A. Ramani, B. Grammaticos and J. Hietarinta, Discrete versions of the Painlevé equations, Phys. Rev. Lett. 67(14) (1991) 1829.

[15] A. Ramani, D. Takahashi, B. Grammaticos and Y. Ohta, The ultimate discretisation of the Painlevé equations, Phys. D 114(3-4) (1998) 185-196.

[16] J. Richter-Gebert, B. Sturmfels and T. Theobald, First steps in tropical geometry, Preprint arXiv:math.AG/0306366.

[17] C. Ormerod, Ultradiscrete connection matrices over a tropical semiring, to appear.

[18] G. R. W. Quispel, H. W. Capel and J. Scully, Piecewise-linear soliton equations and piecewiselinear integrable maps, J. Phys. A 34(11) (2001) 2491-2503.

[19] W. H. Schikhof, Ultrametric calculus: An introduction to p-adic analysis, Cambridge Studies in Advanced Mathematics (Cambridge University Press, Cambridge, 1984), p. 4.

[20] D. Takahashi and J. Satsuma, A soliton cellular automaton, J. Phys. Soc. Japan 59(10) (1990) $3514-3519$.

[21] D. Takahashi, T. Tokihiro, B. Grammaticos, Y. Ohta and A. Ramani, Constructing solutions to the ultradiscrete Painlevé equations, J. Phys. A 30(22) (1997) 7953-7966.

[22] T. Tokihiro, D. Takahashi, J. Matsukidaira and J. Satsuma, From soliton equations to integrable cellular automata through a limiting procedure, Phys. Rev. Lett. 76(18) (1996) 3247-3250. 